\title{
A Case Report of Preoperative, Intraoperative, and Postoperative Anterior Chamber Shallowing Resulting from Different Mechanisms
}

\author{
Yujiro Mori Yoshifumi Ikeda Ichiya Sano Etsuko Fujihara \\ Masaki Tanito \\ Division of Ophthalmology, Matsue Red Cross Hospital, Matsue, Japan
}

\section{Key Words}

Flat anterior chamber - Short axial length - Primary angle closure glaucoma - Acute intraoperative choroidal effusion · Capsular block syndrome · Cataract surgery · Vitrectomy . Neodymium:yttrium-aluminium-garnet laser

\begin{abstract}
A 54-year-old woman with an epiretinal membrane in her left eye accompanied by a shallow anterior chamber due to primary angle closure glaucoma underwent vitrectomy and cataract surgery. During the cataract surgery, immediately after the ultrasonic tip had been removed from the anterior chamber, anterior chamber flattening occurred. An intraoperative fundus examination showed the development of acute intraoperative choroidal effusion. Postoperatively, the anterior chamber remained shallow even after the choroidal detachment had subsided; capsular bag distension seen by ultrasound biomicroscopy suggested the development of early postoperative capsular block syndrome. After neodymium:yttrium-aluminiumgarnet laser capsulotomy, the anterior chamber deepened. Depending on the perioperative period, the mechanism of a flat anterior chamber can change, and understanding the underlying mechanisms is required for appropriate treatment.


Mori et al.: A Case Report of Preoperative, Intraoperative, and Postoperative Anterior Chamber Shallowing Resulting from Different Mechanisms

\section{Introduction}

The anterior chamber can flatten as a result of various mechanisms, although each mechanism is relatively rare, and requires appropriate therapy based on the underlying etiology. We report a case with anterior chamber shallowing observed preoperatively, intraoperatively, and postoperatively resulting from different mechanisms.

\section{Case Presentation}

A 54-year-old woman was referred to our hospital for surgical treatment of an epiretinal membrane in her left eye (OS). Preoperatively, the best-corrected visual acuity was 0.2 with a +4.25-diopter hyperopic correction, and the intraocular pressure (IOP) was $22 \mathrm{~mm} \mathrm{Hg}$ with one ocular hypotensive medication OS. Slit-lamp examination showed a shallow anterior chamber with a van Herick grade I narrow angle (fig. 1a, b); gonioscopy revealed the presence of peripheral anterior synechia at a temporal anterior chamber angle $(<0.5$ clock hour). Ultrasound biomicroscopy (UBM) under mydriasis revealed functional angle closure, while neither uveal effusion nor ciliary detachment was observed in any quadrants preoperatively (fig. 1c), with an UBM-measured horizontal corneal diameter of $11.0 \mathrm{~mm}$ and an Amode-measured axial length of $21.96 \mathrm{~mm}$ OS. The vertical cup-to-disc ratio was 0.8 , with rim notching in the superotemporal region. The patient was diagnosed with a shallow anterior chamber due to primary angle closure glaucoma concomitant with the epiretinal membrane. A combined cataract surgery and vitrectomy was planned to treat both pathologies.

During the cataract surgery, immediately after the ultrasonic tip had been removed from the anterior chamber, anterior chamber flattening occurred; the anterior chamber formation was poor during aspiration of the residual cortex due to anterior bulging of the posterior lens capsule (fig. 1d, arrow). Fundus examination during the vitrectomy showed the development of uveal effusion (fig. 1e, arrow), suggesting that acute intraoperative choroidal effusion (AICE) [1] was the mechanism of the intraoperative anterior chamber flattening. At the end of the surgery, an intraocular lens (IOL) was implanted into the capsular bag.

Postoperatively, the anterior chamber remained shallow even after the choroidal detachment had subsided (fig. 1f); the IOP was $34 \mathrm{~mm} \mathrm{Hg}$ with 4 ocular hypotensive medications OS on postoperative day 6. UBM showed marked distension of the capsular bag and anterior displacement of the IOL and iris plane (fig. 1g, the arrowheads indicate the expanded posterior capsule), suggesting that early postoperative capsular block syndrome (CBS) [2] was the mechanism of the shallowing of the anterior chamber. As a result of neodymium:yttrium-aluminium-garnet laser capsulotomy, the anterior chamber deepened immediately (fig. 1h), and the iris plane and IOL moved posteriorly (fig. 1i).

At the final visit 9 months postoperatively, the best-corrected visual acuity was 0.4 , the IOP was $13 \mathrm{~mm} \mathrm{Hg}$, and the anterior chamber remained deeper compared with preoperatively.

\section{Discussion}

Sudden onset of choroidal detachment and simultaneous anterior chamber flattening observed in the current case are typical findings of AICE. The etiology of AICE has not been fully recognized yet, although the short axial length, narrow angle, and hyperopia, as seen in the current case, are also the generally recognized risk factors for surgery-related choroidal 
Mori et al.: A Case Report of Preoperative, Intraoperative, and Postoperative Anterior Chamber Shallowing Resulting from Different Mechanisms

effusion [3]. Previously, when AICE was encountered, immediate wound closure, subsequent increase in the IOP to more than $80 \mathrm{~mm} \mathrm{Hg}$, and vitrectomy were the recommended therapies [3]. The modern small incisional cataract surgery and vitrectomy may be the reasons for the favorable outcome in the current case. Assessed by UBM, Sakai et al. [4] have found that uveal effusion was present in $14 \%$ of chronic primary angle closure eyes. Accordingly, we could not fully exclude the preoperative presence of uveal effusion in this case.

Depending on the time of onset, CBS can be classified into three forms, including intraoperative CBS seen at the time of lens luxation after hydrodissection, early postoperative CBS that accompanies trapped balanced salt solution toward the posterior capsule, and late postoperative CBS with liquefied aftercataract or lacteocrumenasia [2]. At the end of surgery, after IOL implantation, the viscoelastic material was difficult to remove because of the bulging of the posterior capsule; thus, insufficient removal of viscoelastic material may have been associated with the early postoperative CBS in the current case.

Despite the intraoperative and postoperative complications, we obtained visual recovery, an IOP reduction, and anterior chamber deepening after surgery as reported in primary angle closure glaucoma eyes [5]. Depending on the perioperative period, the mechanism of the flattening of the anterior chamber can change, and, therefore, an understanding of the underlying mechanisms is required for appropriate treatment.

\section{Statement of Ethnics}

Written informed consent was obtained from the patient for publication of this case report and any accompanying images.

\section{Disclosure Statement}

The authors declare that there is no conflict of interest regarding the publication of this paper.

\section{References}

1 Maumenee AE, Schwartz MF: Acute intraoperative choroidal effusion. Am J Ophthalmol 1985;100:147-154.

-2 Miyake K, Ota I, Ichihashi S, Miyake S, Tanaka Y, Terasaki H: New classification of capsular block syndrome. J Cataract Refract Surg 1998;24:1230-1234.

-3 Wu W, Dawson DG, Sugar A, Elner SG, Meyer KA, McKey JB, Moroi SE: Cataract surgery in patients with nanophthalmos: results and complications. J Cataract Refract Surg 2004;30:584-590.

4 Sakai H, Morine-Shinjyo S, Shinzato M, Nakamura Y, Sakai M, Sawaguchi S: Uveal effusion in primary angleclosure glaucoma. Ophthalmology 2005;112:413-419.

5 Omura T, Tanito M, Doi R, Ishida R, Ohira A: Anterior chamber parameters measured using the Pentacam Scheimpflug imaging device before and after cataract surgery in eyes with primary angle closure. Acta Ophthalmol 2012;90:e654-e655. 


\section{Case Reports in \\ Ophthalmology}

\begin{tabular}{l|l}
\hline Case Rep Ophthalmol 2016;7:115-118 \\
\hline DOI: 10.1159/000444215 & $\begin{array}{l}\text { @ } 2016 \text { The Author(s). Published by S. Karger AG, Basel } \\
\text { www.karger.com/cop }\end{array}$ \\
\hline
\end{tabular}

Mori et al.: A Case Report of Preoperative, Intraoperative, and Postoperative Anterio Chamber Shallowing Resulting from Different Mechanisms
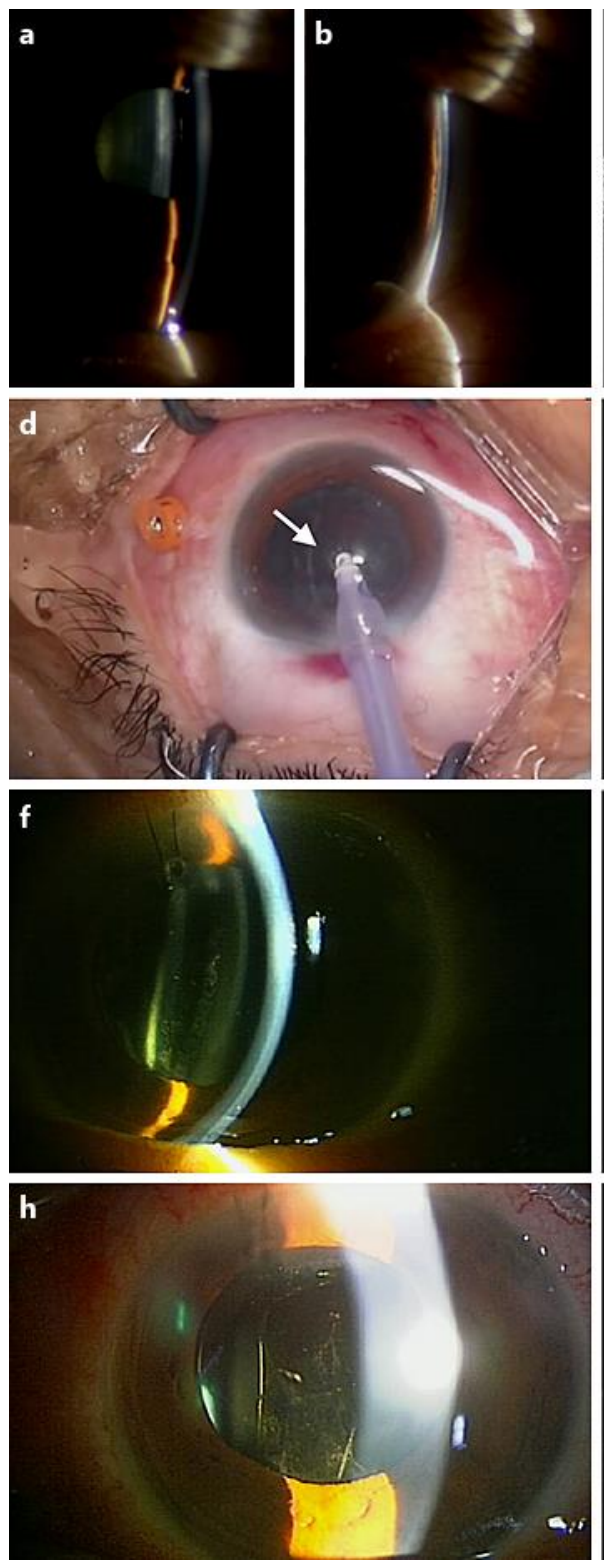

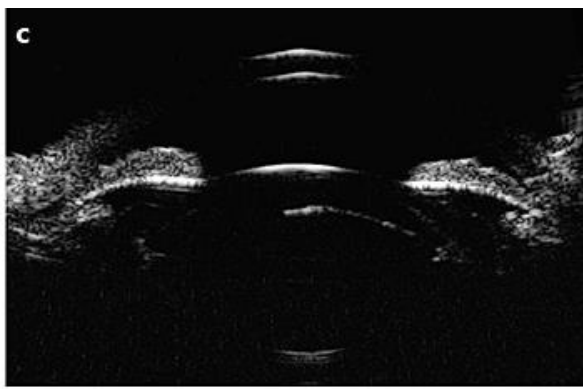

e
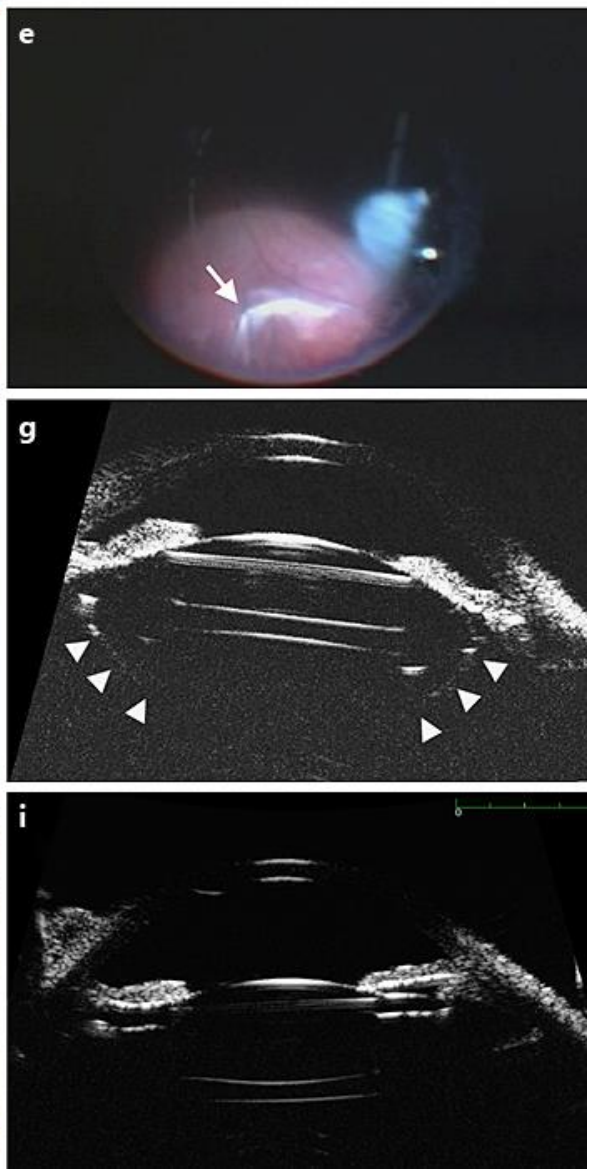

Fig. 1. Perioperative findings. Preoperatively, a shallow anterior chamber is observed at the center (a) and periphery (b); UBM shows functional angle closure (c). Intraoperatively, difficulty with anterior chamber formation due to forward bulging of the posterior lens capsule (arrow) is encountered (d); acute uveal effusion is observed during vitrectomy (e, arrow). Postoperatively, the anterior chamber is shallow even after the uveal effusion resolved ( $f$, postoperative day 6); UBM shows forward displacement of the iris plane and IOL due to expansion of the capsular bag $(\mathbf{g}$, the arrowheads indicate the expanded posterior capsule). After the capsulotomy, the anterior chamber deepened (h) and anterior displacement of the iris plane and IOL resolved (i). 